\title{
Mortality trends among people with hepatitis B and C: a population-based linkage study, 1993-2012
}

Maryam Alavi ${ }^{1 *} \mathbb{D}$, Jason Grebely ${ }^{1}$, Behzad Hajarizadeh', Janaki Amin,2, Sarah Larney ${ }^{3}$, Matthew G. Law ${ }^{1}$, Jacob George ${ }^{4}$, Louisa Degenhardt ${ }^{3}$ and Gregory J. Dore ${ }^{1}$

\begin{abstract}
Background: This study evaluated cause-specific mortality trends including liver-related mortality among people with a hepatitis B virus (HBV) and hepatitis C virus (HCV) notification in New South Wales, Australia.

Methods: Notifications 1993-2012 were linked to cause-specific mortality records 1993-2013.

Results: Among 57,929 and 92,474 people with a HBV and HCV notification, 4.8\% and 10.0\% died since 1997. In early 2010 s, $28 \%$ and $33 \%$ of HBV and HCV deaths were liver-related, $28 \%$ and $17 \%$ were cancer-related (excluding liver cancer), and 5\% and 15\% were drug-related, respectively. During 2002-2012, annual HBV-related liver death numbers were relatively stable (53 to 68), while HCV-related liver death numbers increased considerably (111 to 284). Age-standardised HBV-related liver mortality rates declined from 0.2 to 0.1 per 100 person-years (PY) $(P<0.001)$; however, HCV-related rates remained stable $(0.2$ to 0.3 per $100 \mathrm{PY}, P=0.619)$. In adjusted analyses, older age was the strongest predictor of liver-related mortality [birth earlier than 1945, HBV adjusted hazard ratio (aHR) 28.1, 95\% Cl 21.0, 37.5 and; HCV aHR 31.9, 95\% Cl 26.8, 37.9], followed by history of alcohol-use disorder (HBV aHR 7.0, 95\% Cl 5.5, 8.8 and; HCV aHR 8.3, 95\% Cl 7.6, 9.1).

Conclusions: Declining HBV-related liver mortality rates and stable burden suggest an impact of improved antiviral therapy efficacy and uptake. In contrast, the impact of interferon-containing HCV treatment programs on liver-related mortality individual-level risk and population-level burden has been limited. These findings also highlight the importance of HBV/HCV public health interventions that incorporate increased antiviral therapy uptake, and action on health risk behaviors.
\end{abstract}

Keywords: HBV, HCV, Cause-specific mortality, Drug-related mortality, Liver-related mortality

\section{Background}

The global burden of chronic hepatitis B virus (HBV) and hepatitis $\mathrm{C}$ virus (HCV) infections is rising, with $\mathrm{HBV}$ and $\mathrm{HCV}$-related deaths increasing from 0.8 to 1.4 million over the period 1990 to 2013 [1]. Ageing populations with chronic HBV and HCV and limited uptake of effective antiviral therapy are driving this burden. Therapeutic advances, particularly the advent of direct-acting antiviral (DAA) therapy for chronic $\mathrm{HCV}$, provide optimism that major reductions in population-level liver-related mortality are

\footnotetext{
* Correspondence: msalehialavi@kirby.unsw.edu.au

${ }^{1}$ Biostatistics and Databases Program, The Kirby Institute, UNSW Sydney,

Wallace Wurth Building, UNSW, Sydney, NSW 2052, Australia

Full list of author information is available at the end of the article
}

achievable [2]. In fact, the World Health Organization (WHO) has recently set a target of a $65 \%$ reduction in HBV- and HCV-related mortality by 2030 [3]. In this context, ongoing surveillance of mortality trends is crucial to evaluate the efficacy of implementation of public health strategies against these infections.

Evaluation of mortality among people with HBV and $\mathrm{HCV}$ should ideally involve characterisation of causespecific mortality. Co-morbidities are particularly prevalent among $\mathrm{HCV}$ populations, related to ongoing drug and alcohol use [4, 5], extrahepatic manifestations of HCV [6], or ageing-related chronic disorders [7]. Even within liverrelated mortality there may be differential trends for decompensated cirrhosis (DC) and hepatocellular carcinoma

(c) The Author(s). 2018 Open Access This article is distributed under the terms of the Creative Commons Attribution 4.0 International License (http://creativecommons.org/licenses/by/4.0/), which permits unrestricted use, distribution, and 
(HCC) [8]. Characterisation of these mortality patterns before the rapid scale-up of DAA therapies is a public health priority, providing a foundation for the evaluation of the impact of new treatments on the disease burden of HCV.

Globally, Australia is among the few settings with established national surveillance systems that enable ongoing monitoring of all people notified with $\mathrm{HBV}$ and $\mathrm{HCV}$ infections, by linking notifications, hospitalisation, and mortality databases. The aim of this study was to assess cause-specific mortality trends and liver-related mortality risk factors among people with an HBV and HCV notification in New South Wales (NSW), Australia.

\section{Methods}

\section{Study population and data sources}

The study population consisted of all people recorded in the NSW Notifiable Conditions Information Management System (NCIMS) with an HBV and HCV notification. NSW NCIMS holds records of all individuals with positive HBV and HCV serology tests, notified of diagnoses via mandatory notification procedures, since 1991 [9]. Notifiable $\mathrm{HBV}$ and $\mathrm{HCV}$ cases require detection of $\mathrm{HBV}$ surface antigen or HBV DNA and anti-HCV antibody or HCV RNA, respectively.

People with HIV co-infection were identified through the National HIV Registry (NHR). NHR receives all notifications of HIV infection in Australia via mandatory notification procedures, since 1985 [10].

Deceased people were identified through the NSW Registry of Births, Deaths and Marriages (RBDM) and Cause of Death Unit Record File (COD URF). All deaths in NSW are registered within the NSW RBDM (since 1993) and coded causes are held within the COD URF. Since 1997, deaths are coded using the 10th revision of the International Classification of Diseases and Related Health Problems (ICD-10) [9].

Hospital admissions for alcohol-use disorder (AUD) and end-stage liver disease (ESLD) were identified through the NSW Admitted Patient Data Collection (APDC). Since 2001, NSW APDC holds inpatient admission information from all NSW hospitals, including diagnosis information coded at the time of discharge by ICD-10 [9]. People with a history of opioid substitution therapy (OST) were identified through the Pharmaceutical Drugs of Addiction System (PHDAS). PHDAS is used by the NSW Ministry of Health to issue authorities for applicant prescribers to prescribe drugs of addiction, including methadone (since 1985) and buprenorphine (since 2001) under the NSW Opioid Treatment Program [9].

\section{Data linkage}

Data linkage occurred in two stages. First, NSW NCIMS HBV and HCV notifications were matched internally, to identify people with co-infection. Second, records were linked deterministically and probabilistically between the NSW NCIMS and NHR, and the NSW NCIMS and APDC, PHDAS, and RBDM and COD URF, respectively. Demographic details used for record linkages included full name, name codes (only for NHR), gender, date of birth, and address. NSW Centre for Health Record Linkage undertook all the linkages. Probabilistic linkage procedures had an error rate of $0.5 \%$ or less (i.e. false positive and false negative rates of 5/1000) [9].

\section{Study period}

$\mathrm{HBV}$ and HCV notifications and mortality records were extracted for the study period between 1 January 1993 and 31 December 2012, 1 January 1993 and 31 December 2013, respectively.

\section{Study outcome}

The primary outcome of interest was liver-related mortality, defined by multiple causes of death (i.e. coded in the underlying and/or contributing fields of a linked record, Additional file 1: Table S1). To improve the accuracy of this definition, contributing fields were included only if a prior ESLD-related hospitalisation was documented (defined by DC and/or HCC admissions, Additional file 1: Table S1). This definition was selected after considering a number of different classifications. Compared to those excluding secondary causes, a multiple-cause definition was believed to complement the routine description of liver-related mortality that would use only the underlying cause [11]. In the sensitivity analyses, trends of liver-related mortality were evaluated using three alternative definitions, including underlying and/or contributing causes of death (definition 2); underlying causes (definition 3); and underlying causes with prior ESLD admission (definition 4).

\section{Exclusion criteria}

Records where date of death was prior to introduction of ICD-10 coding (calendar year 1997), and records where the date of $\mathrm{HBV}$ or $\mathrm{HCV}$ notification occurred after censoring were excluded.

\section{Statistical analysis}

Among people with an HBV and HCV notification, cause-specific mortality numbers were first described by chapters of ICD-10. Liver-related mortality numbers were compared between four definitions, defined over several chapters of ICD-10, and ranging from the most conservative (definition 4) to the least conservative estimates (definition 2). Trends in cause-specific mortality (liver-, circulatory system-, drug-, and cancer-related, excluding liver cancer) numbers, incidence rates, and agestandardised incidence rates [per 100 person-years (PY)] were evaluated. The Australian Standard Population 
2001 was used for standardisation. The strength of the association between risk factors and liver-related mortality was assessed by unadjusted and adjusted Cox proportional hazards regressions. Risk factors included birth cohort, gender, country of birth, calendar period of hepatitis notification, $\mathrm{HBV} / \mathrm{HCV} / \mathrm{HIV}$ co-infection, geographical area of residence at the time of hepatitis notification, history of AUD, and history of OST. Following unadjusted analyses, multivariable regressions were performed to evaluate factors associated with liver-related mortality, considering factors significant at the 0.20 level in the unadjusted models.

A multiple-cause definition was not used for nonliver-related causes of death (circulatory system-, drug-, and cancer-related); i.e. in all analyses, only the underlying field of a linked record was used to define these deaths (Additional file 1: Table S1). Other cause-related mortality was defined excluding ICD-10 codes used in the definitions of liver-, circulatory system-, drug, and cancer-related mortality (excluding liver cancer). AUD was the label used to define continued drinking despite adverse mental and physical consequences [12], and having at least one AUD-related hospital admission was referred to as history of AUD. AUD-related admissions were defined using the primary and/or secondary fields of linked hospitalisation records (Additional file 1: Table S1). In all analysis, having at least one episode of OST was referred to as history of OST.

To calculate all-cause and ICD-10 chapter-specific mortality numbers, all linked mortality records between 1997 and 2013 were included in analyses (including deaths that occurred within 6 months post the date of HCV notification). To calculate cause-specific mortality numbers and rates (liver-, circulatory system-, drug-, and cancer-related), and assess factors associated with liver-related mortality; linked mortality records between 2002 and 2013 were included in analyses. In all survival analyses, linked mortality records prior to 1 January 2002 were censored, given availability of hospitalisation data since 2001 (required to define liver-related mortality definition numbers one and four). In all survival analyses, people with a missing date of birth were excluded. Finally, in all survival analyses, observation time was defined to start 6 months post the date of HCV notification [13], and to end at whichever occurred first; death, or end of follow-up. Statistical analyses were carried out in STATA versions 12.

\section{Results}

\section{Study population}

During 1993-2012, 57,929 and 92,474 people in NSW had an HBV and HCV notification, respectively. Since 1997 (use of ICD-10 codes), $4.8 \%$ and $10.0 \%$ of the populations with an $\mathrm{HBV}$ and $\mathrm{HCV}$ notification died, respectively. Among those who had died, $42 \%$ and 52\% were born during $1945-1965,75 \%$ and $71 \%$ were male, $3 \%$ and $2 \%$ had HIV co-infection, $13 \%$ and $29 \%$ had a history of AUD, and $8 \%$ and $31 \%$ had a history of OST, respectively. Among people with an $\mathrm{HBV}$ notification, $17 \%$ of the deceased had $\mathrm{HBV} / \mathrm{HCV}$ co-infection (Table 1).

Infectious and parasitic diseases comprised 10\% of HBV- and HCV-related deaths. Among people with an HBV notification, neoplasms were the leading cause of mortality (44\%), followed by diseases of the circulatory system (17\%). External causes of death and neoplasms were the first and second most frequently recorded causes of HCV-related mortality (25\% and $24 \%$, respectively) (Additional file 1: Table S2).

\section{Liver-related mortality, definitions}

By the most conservative definition (definition 4), during 2002-2012, HBV- and HCV-related liver mortality numbers increased from 39 to 56 and 74 to 208 , respectively. Defined by multiple causes of death, during 2002-2012, $\mathrm{HBV}$ - and HCV-related liver mortality numbers rose from 53 to 68 and 111 to 284, respectively (Fig. 1).

\section{Cause-specific mortality, age-specific and age-standardized rates}

During 2002-2012, among people with an HBV notification, age-standardised rates of liver-related mortality $(n=582)$ declined from 0.2 to 0.1 per $100 \mathrm{PY}(P<0.001)$ (Fig. 2). Incidence rates of liver-, circulatory system- $(n=340)$, and cancer-related deaths $(n=502)$ increased with increasing age, noticeably among people born during 1955-1964 and earlier (Fig. 3).

During 2002-2012, among people with an HCV notification, age-standardised rates of liver-related mortality $(n=2215)$ remained stable, 0.2 to 0.3 per 100 PY, $P=0.619$. Age-standardised rates of circulatory system-related mortality $(n=985)$ lowered from 0.2 to 0.1 per $100 \mathrm{PY}, P=0.026$. However, rates of drug-related mortality $(n=1110)$ increased during this period, from 0.1 to 0.2 per 100 PY, $P<0.001$ (Fig. 2). Incidence rates of liverand cancer-related deaths $(n=1072)$ increased with increasing age, markedly among people born during 19551964 and earlier. However, drug-related mortality rates were highest among people born in 1975 or later, and lower among older age groups (Fig. 3).

\section{Liver-related mortality, associated factors}

In unadjusted analysis, HBV-related liver mortality was associated with older age, male gender, $\mathrm{HCV}$ coinfection, HIV co-infection, history of AUD and history of OST. People born in Asia-Pacific and those residing in outer metropolitan and metropolitan areas of NSW at the time of HBV notification had reduced liver-related 
Table 1 Demographic characteristics among people with an HBV and HCV notification, NSW 1993-2012, $n=150,403$

\begin{tabular}{|c|c|c|c|c|c|c|c|c|}
\hline \multirow[t]{3}{*}{ Characteristics, n \% } & \multicolumn{4}{|c|}{$\mathrm{HBV}, n=57,929$} & \multicolumn{4}{|c|}{$\mathrm{HCV}, n=92,474$} \\
\hline & \multicolumn{2}{|l|}{ Alive } & \multicolumn{2}{|l|}{ Deceased $^{a}$} & \multicolumn{2}{|l|}{ Alive } & \multicolumn{2}{|l|}{ Deceased $^{a}$} \\
\hline & $n=55,147$ & $\%$ & $n=2782$ & $\%$ & $n=83,267$ & $\%$ & $n=9207$ & $\%$ \\
\hline \multicolumn{9}{|l|}{ Birth cohort $^{b}$} \\
\hline$\geq 1965$ & 31,000 & 56 & 423 & 15 & 43,919 & 53 & 2253 & 24 \\
\hline 1945-1964 & 20,595 & 37 & 1166 & 42 & 35,964 & 43 & 4795 & 52 \\
\hline$\leq 1944$ & 3541 & 6 & 1193 & 43 & 3359 & 4 & 2159 & 23 \\
\hline Male gender ${ }^{\mathrm{b}}$ & 29,949 & 55 & 2074 & 75 & 51,062 & 62 & 6507 & 71 \\
\hline \multicolumn{9}{|l|}{ Country of birth ${ }^{b}$} \\
\hline Australia & 5406 & 20 & 546 & 30 & 34,263 & 78 & 4030 & 71 \\
\hline Asia-Pacific & 16,692 & 63 & 817 & 45 & 4037 & 9 & 490 & 9 \\
\hline Middle East and North Africa & 2029 & 8 & 85 & 5 & 1263 & 3 & 198 & 4 \\
\hline Africa, excluding North Africa & 577 & 2 & 14 & 1 & 191 & $<1$ & 15 & $<1$ \\
\hline Europe & 1782 & 7 & 322 & 18 & 3512 & 8 & 884 & 16 \\
\hline Americas & 134 & 1 & 11 & 1 & 415 & 1 & 39 & 1 \\
\hline \multicolumn{9}{|l|}{ Year of viral hepatitis notification } \\
\hline$\leq 2000$ & 24,696 & 45 & 1634 & 59 & 43,067 & 52 & 6014 & 65 \\
\hline $2001-2006$ & 16,514 & 30 & 782 & 28 & 23,250 & 28 & 2229 & 24 \\
\hline $2007-2012$ & 13,937 & 25 & 366 & 13 & 16,950 & 20 & 964 & 10 \\
\hline HBV/HCV co-infection ${ }^{c}$ & 3232 & 6 & 480 & 17 & - & - & - & - \\
\hline HIV co-infection & 276 & 1 & 72 & 3 & 812 & 1 & 153 & 2 \\
\hline HBV/HCV/HIV co-infection ${ }^{c}$ & 50 & $<1$ & 14 & $<1$ & - & - & - & - \\
\hline \multicolumn{9}{|c|}{ Area of residence at the time of viral hepatitis notification ${ }^{b, d}$} \\
\hline Rural & 5342 & 10 & 440 & 16 & 28,610 & 35 & 2963 & 32 \\
\hline Outer metropolitan & 25,068 & 46 & 1245 & 45 & 26,608 & 33 & 3167 & 35 \\
\hline Metropolitan & 24,183 & 44 & 1082 & 39 & 26,331 & 32 & 3025 & 33 \\
\hline History of alcohol-use disorder & 1566 & 3 & 374 & 13 & 12,855 & 15 & 2652 & 29 \\
\hline History of OST & 1442 & 3 & 225 & 8 & 24,427 & 29 & 2813 & 31 \\
\hline
\end{tabular}

${ }^{a}$ Mortality numbers included during the ICD-10 era, 1997-2013, bamong people with available information: $n=36$ and $n=25$ had missing date of birth among those with an HBV and HCV notifications, respectively; $n=500$ and $n=341$ had missing gender among those with an HBV and HCV notifications, respectively; $n=29,514$ and $n=43,137$ had missing country of birth among those with an HBV and HCV notifications, respectively and; $n=569$ and $n=1718$ had missing area of residence at the time of HBV and HCV notifications, respectively, ${ }^{c}$ for description of baseline characteristics, HBV/HCV and HBV/HCV/HIV co-infection cases were only included among people with an HBV notification, dgeographical area of residence was defined by the 2011 local health district boundaries in NSW, including eight areas covering metropolitan regions and seven covering rural and regional locations

mortality risk (Additional file 1: Table S3). In adjusted analysis, HBV-related liver mortality was associated with older age (birth earlier than 1945, and during 1945-1965), male gender, more recent periods of $\mathrm{HBV}$ notification years (2001-2006, and 2007-2012), HCV co-infection, HIV co-infection, and history of AUD (Table 2).

In unadjusted analysis, HCV-related liver mortality was associated with older age, male gender, more recent periods of HCV notification, HBV co-infection, HIV co-infection and history of AUD. People with a history of OST had reduced liver-related mortality risk (Additional file 1: Table S4). In adjusted analysis, HCV-related liver mortality was associated with older age (birth earlier than 1945, and during 1945-1965), male gender, recent periods of
HCV notification (2001-2006, and 2007-2012), HBV coinfection, HIV co-infection, and history of AUD (Table 2).

\section{Cause-specific mortality, temporal trends}

Among people with an HBV notification, DC-related mortality decreased from $10 \%$ of all causes in 2002-2004 to $6 \%$ in $2011-2013(P<0.001)$; however, proportions of HCC-related mortality remained stable during this period $(18 \%$ and $16 \%$ of all causes, $P=0.692)$. In the early 2010s, other cancers and diseases of the circulatory system comprised $28 \%$ and $17 \%$ of all deaths, respectively (Fig. 4).

Among people with an $\mathrm{HCV}$ notification, DC-related mortality comprised $10 \%$ of all causes in 2002-2004 and 

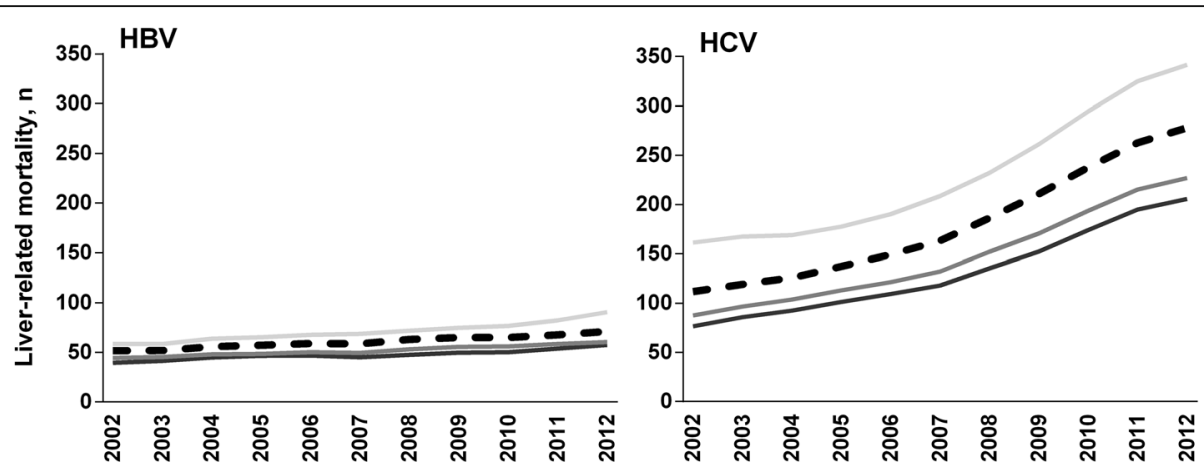

definition 1, by underlying and/or contributing causes of death, if contributing causes were linked to a prior ESLD admission

- definition 2, by underlying and/or contributing causes of death

- definition 3 , by the underlying cause of death

- definition 4 , by the underlying cause of death, if linked to a prior ESLD admission

Fig. 1 Liver-related mortality numbers and definitions among people with an HBV and HCV notification, NSW 1993-2012, n=150,403

2011-2013 ( $P=0.277)$; however, HCC-related mortality increased from $6 \%$ to $10 \%$ during this period $(P<0.001)$. In the early $2010 \mathrm{~s}$, other cancers and drug-related causes comprised $17 \%$ and $15 \%$ of all deaths, respectively (Fig. 4).

\section{Other cause-related mortality}

Among people with an HBV notification, there were 620 other cause-related deaths. The majority of these deaths occurred among people born in 1954 or earlier $(60 \%, n=370)$ and were most frequently due to infectious and parasitic diseases (17\%, 63 of 370). Among people with an HCV notification, there were 2298 other cause-related deaths. The majority of these deaths occurred among people born in 1955 or later $(61 \%, n=1397)$ and were most frequently due to external causes $(59 \%, n=821)$, including intentional self-harm by hanging, strangulation, and suffocation (28\%, 232 of 821$)$, other self-harm $(16 \%, 134$ of 821$)$, and transport accidents (20\%, 165 of 821 ) (Additional file 1: Table S5).

\section{Discussion}

This study demonstrated opposing trends in liver-related mortality among people with an HBV and HCV notification in NSW, Australia. During 2002-2012, among people with HBV age-standardised risk of liver mortality has reduced significantly, while the population-level burden of liver mortality (total deaths per year) has remained relatively stable. By contrast, among people with $\mathrm{HCV}$ age-standardised risk of liver mortality has remained relatively stable, while population-level burden has markedly increased. The potential impact of improving HBV antiviral therapy since the mid-2000s is encouraging; however, these trends underline the relatively limited impact of interferon-based $\mathrm{HCV}$ treatment.
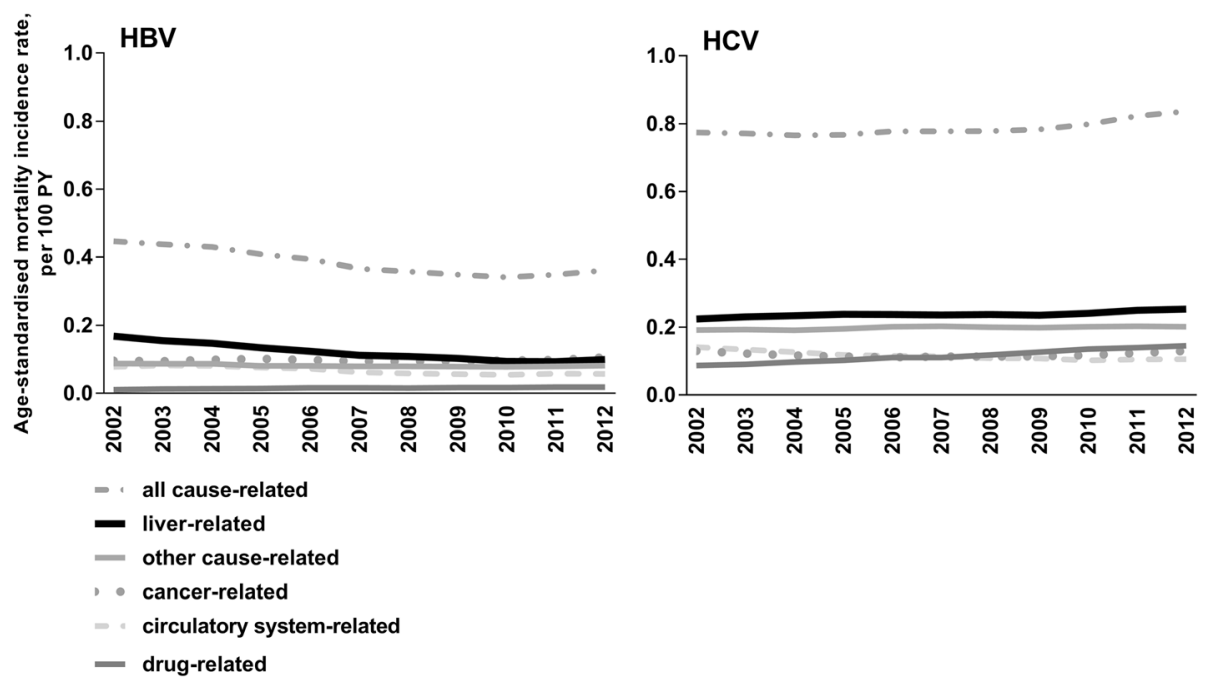

Fig. 2 Age-standardised mortality incidence rates among people with an HBV and HCV notification, NSW 1993-2012, n=150,403 

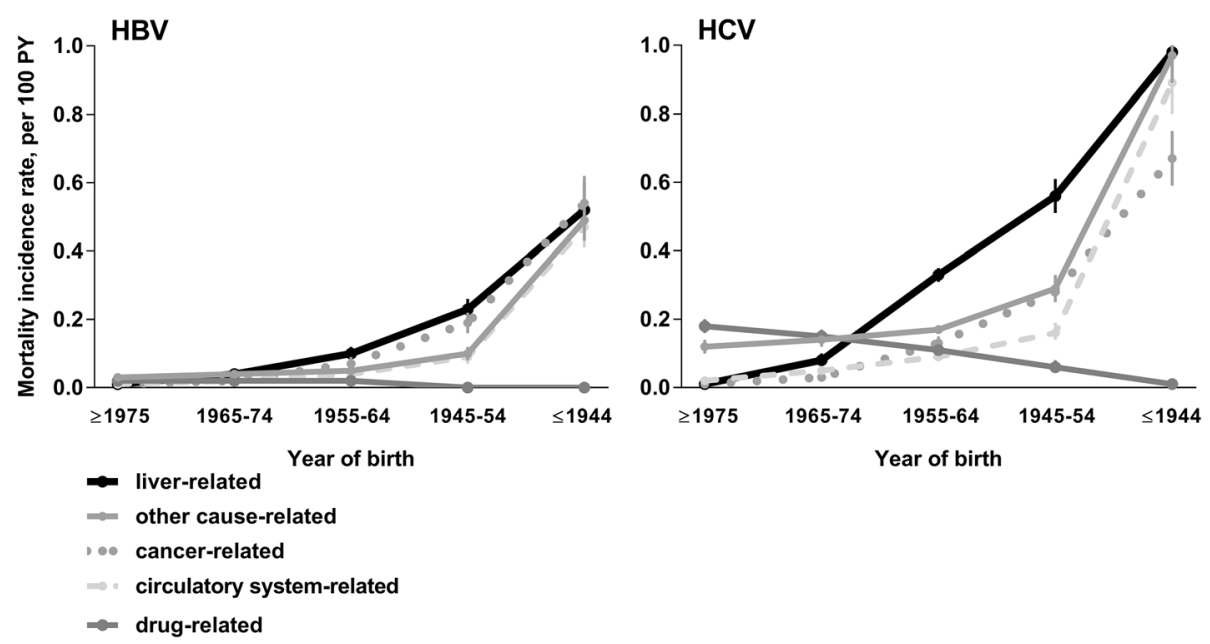

Fig. 3 Cause-specific mortality incidence rates among people with an HBV and HCV notification, NSW 1993-2012, by year of birth, $n=150,403$

Non-liver-related causes comprised the majority of HBV and $\mathrm{HCV}$ deaths throughout the study period, highlighting the need for a comprehensive strategy for reducing morbidity and mortality among people with HBV and $\mathrm{HCV}$. Mandatory HBV and HCV notifications, availability of this data for research, and the capacity for regular linkages to other routinely collected administrative databases provides the opportunity for ongoing evaluation of HBV and $\mathrm{HCV}$ public health strategies, including the introduction of government-subsidized broad access to interferonfree DAA HCV treatment in Australia from 2016.

Among people with an HBV notification, proportions of DC-related mortality significantly declined between early 2000s and early 2010s; however, HCC-related mortality remained stable during this period. Improved HBV therapies have been shown to reduce the risks of hepatic events, HCC, liver-related, and all-cause mortality, particularly among people with cirrhosis [14]. However, current regimens cannot completely prevent $\mathrm{HCC}$, and regular surveillance is still required in at-risk groups (predominantly those with cirrhosis) even when HBV DNA is undetectable [15]. In Australia, the number of people receiving HBV treatment has increased in recent years, from 8500 in 2006 to 11,000 in 2012 [16, 17]; however, substantial gaps remain in the HBV cascade of care, given that up to two-thirds of eligible individuals are not receiving therapy [17]. The potential to further reduce the mortality burden of HBV infection through enhanced diagnosis and treatment uptake is considerable.

Among people with an $\mathrm{HCV}$ notification, proportions of DC-related mortality were stable between early $2000 \mathrm{~s}$ and early 2010s, while HCC-related mortality increased in this period. Despite improvements in $\mathrm{HCV}$ antiviral therapy, small numbers of individuals were treated during 2000s-early 2010s, and treatment outcomes were sub-optimal [18]. Moving forward, a combination of enhanced treatment efficacy and increased treatment uptake is expected to have a greater impact on HCVrelated mortality [2]. In Australia, access to highly effective interferon-free DAA therapies has been provided via government subsidy from March 2016, regardless of disease stage or drug and alcohol use. This has enabled a rapid initial uptake of DAA therapy [19], with the potential to reduce liver-related mortality. Importantly, entry into $\mathrm{HCV}$ care through diverse models including primary care and drug and alcohol services could improve engagement and retention along the $\mathrm{HCV}$ care cascade and optimise co-morbidity management [20].

More recent notification periods (2001-2006, 20072012) were associated with increased risk of HBV and HCV liver-related mortality, an association that is likely to be driven by age (Additional file 1: Table S6). Other factors associated with liver-related mortality included older age, male gender, $\mathrm{HBV} / \mathrm{HCV} / \mathrm{HIV}$ co-infection, and history of AUD. Given the accelerated progression to advanced liver disease [21-23], where appropriate, these characteristics could be used to constitute highrisk groups that may need to be prioritised in the era of improved antiviral therapies.

Drug-related mortality remained a major cause of death among people with an HCV notification during 2002-2012, which is not surprising given that the majority of $\mathrm{HCV}$ transmissions are among people who inject drugs. In Australia, this period was characterised by shifting heroin and methamphetamine markets, changing patterns of drug use (including poly drug use), and increases in extra-medical opioid use [24-27]. In this context, the rising individual-level risk of drug-related mortality underlines the need for broader access to harm reduction and treatment programs that are responsive to the changing needs of people who use drugs. In addition to mortality from direct effects of drug use, this study 
Table 2 Adjusted analysis of factors associated with liver-related mortality among people with an HBV and HCV notification, NSW 1993-2012, $n=150,403$

\begin{tabular}{|c|c|c|c|c|c|c|c|c|c|c|}
\hline \multirow[t]{3}{*}{ Characteristics, n \% } & \multicolumn{5}{|l|}{ HBV } & \multicolumn{5}{|l|}{$\mathrm{HCV}$} \\
\hline & \multicolumn{2}{|c|}{ Deceased $^{a}$} & \multirow[t]{2}{*}{$a R^{b}$} & \multirow[t]{2}{*}{$95 \% \mathrm{Cl}$} & \multirow[t]{2}{*}{$P$} & \multicolumn{2}{|l|}{ Deceased $^{a}$} & \multirow[t]{2}{*}{$a \mathrm{HR}^{\mathrm{b}}$} & \multirow[t]{2}{*}{$95 \% \mathrm{Cl}$} & \multirow[t]{2}{*}{$P$} \\
\hline & $n=582$ & $\%$ & & & & $n=2215$ & $\%$ & & & \\
\hline \multicolumn{11}{|l|}{ Birth cohort } \\
\hline$\geq 1965$ & 66 & $<1$ & 1.00 & - & - & 247 & 1 & 1.00 & - & - \\
\hline 1945-1964 & 304 & 1 & 6.19 & $4.72,8.11$ & $<0.001$ & 1542 & 4 & 7.33 & $6.39,8.40$ & $<0.001$ \\
\hline$\leq 1944$ & 212 & 5 & 28.07 & $21.02,37.47$ & $<0.001$ & 426 & 9 & 31.88 & $26.83,37.87$ & $<0.001$ \\
\hline \multicolumn{11}{|l|}{ Gender } \\
\hline Female & 102 & $<1$ & 1.00 & - & - & 559 & 2 & 1.00 & - & - \\
\hline Male & 477 & 2 & 2.65 & $2.13,3.30$ & $<0.001$ & 1654 & 3 & 1.47 & $1.33,1.62$ & $<0.001$ \\
\hline Missing & 3 & 1 & 1.44 & $0.45,4.54$ & 0.539 & 2 & 1 & 0.41 & $0.10,1.64$ & 0.208 \\
\hline \multicolumn{11}{|l|}{ Country of birth ${ }^{c}$} \\
\hline Australia & 109 & 2 & 1.00 & - & - & 1024 & 3 & 1.00 & - & - \\
\hline Asia-Pacific & 148 & 1 & 0.89 & $0.67,1.19$ & 0.425 & 130 & 3 & 1.07 & $0.88,1.29$ & 0.493 \\
\hline Other & 76 & 2 & 0.91 & $0.67,1.25$ & 0.580 & 311 & 5 & 1.43 & $1.25,1.63$ & $<0.001$ \\
\hline Missing & 249 & 1 & 0.86 & $0.66,1.12$ & 0.268 & 750 & 2 & 0.88 & $0.79,0.97$ & 0.015 \\
\hline \multicolumn{11}{|l|}{ Year of notification } \\
\hline$\leq 2000$ & 316 & 1 & 1.00 & - & - & 1347 & 3 & 1.00 & - & - \\
\hline $2001-2006$ & 206 & 1 & 1.26 & $1.01,1.58$ & 0.041 & 627 & 2 & 1.48 & $1.32,1.67$ & $<0.001$ \\
\hline 2007-2012 & 60 & $<1$ & 1.44 & $1.01,2.06$ & 0.045 & 241 & 1 & 2.98 & $2.47,3.60$ & $<0.001$ \\
\hline \multicolumn{11}{|l|}{ HBV/HCV co-infection } \\
\hline No & 461 & 1 & 1.00 & - & - & 2094 & 2 & 1.00 & - & - \\
\hline Yes & 121 & 3 & 2.63 & $2.03,3.41$ & $<0.001$ & 121 & 3 & 1.53 & $1.28,1.85$ & $<0.001$ \\
\hline \multicolumn{11}{|l|}{ HIV co-infection } \\
\hline No & 572 & 1 & 1.00 & - & - & 2186 & 2 & 1.00 & - & - \\
\hline Yes & 10 & 3 & 3.46 & $1.83,6.55$ & $<0.001$ & 29 & 3 & 1.78 & $1.23,2.57$ & 0.002 \\
\hline \multicolumn{11}{|c|}{ Area of residence at the time of notification } \\
\hline Rural & 92 & 2 & 1.00 & - & - & - & - & - & - & - \\
\hline Outer metropolitan & 262 & 1 & 1.02 & $0.78,1.32$ & 0.899 & - & - & - & - & - \\
\hline Metropolitan & 227 & 1 & 0.84 & $0.65,1.10$ & 0.207 & - & - & - & - & - \\
\hline Missing & 1 & $<1$ & 0.21 & $0.03,1.53$ & 0.124 & - & - & - & - & - \\
\hline \multicolumn{11}{|c|}{ History of alcohol-use disorder } \\
\hline No & 448 & 1 & 1.00 & - & - & 1050 & 1 & 1.00 & - & - \\
\hline Yes & 134 & 7 & 6.98 & $5.54,8.78$ & $<0.001$ & 1165 & 7 & 8.30 & $7.56,9.10$ & $<0.001$ \\
\hline \multicolumn{11}{|l|}{ History of OST ${ }^{d}$} \\
\hline No & 538 & 1 & 1.00 & - & - & 1675 & 3 & 1.00 & - & - \\
\hline Yes & 44 & 3 & 0.84 & $0.57,1.25$ & 0.391 & 540 & 2 & 0.92 & $0.82,1.03$ & 0.150 \\
\hline
\end{tabular}

${ }^{a}$ Records included 2002-2013, badjusted hazard ratio, ' majority of countries included in the other category were European (HBV $n=50, \mathrm{HCV} n=229$ ).

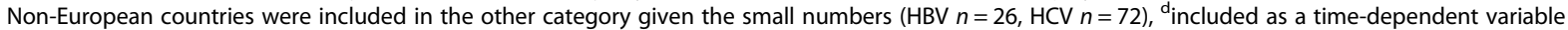

showed a substantial number of deaths among people with $\mathrm{HCV}$ are due to suicide and accidental injuries. These results were sobering, in highlighting the importance of a multidisciplinary response to $\mathrm{HCV}$ that encompasses not only provision of antiviral therapy, but also strategies to improve mental health- and substance use-related outcomes [28].

As populations with $\mathrm{HBV}$ and $\mathrm{HCV}$ age, mortality from non-communicable diseases increases in prominence [7]. Circulatory system-related mortality remained 

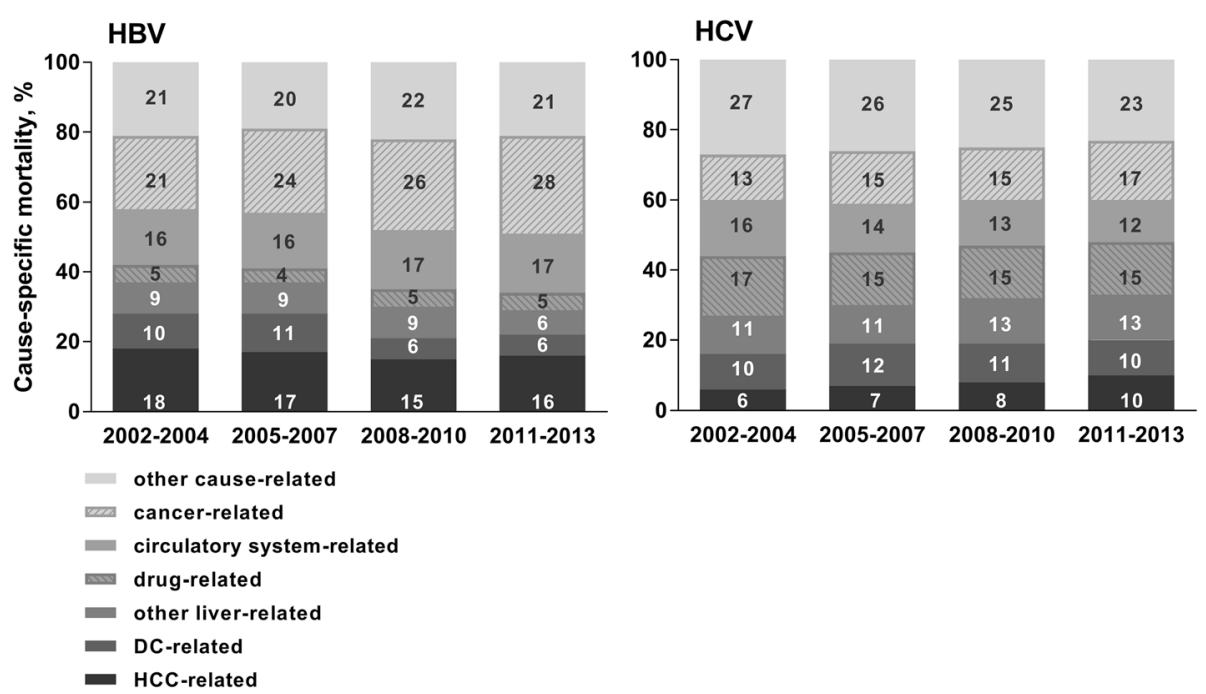

Fig. 4 Percentage of deaths due to different underlying causes among people with an HBV and HCV notification, NSW 1993-2012, n=150,403

a major cause of HBV and HCV deaths in early 2010s; however, declines in its individual-level risk (particularly among people with an HCV notification) is consistent with trends in other high income countries [29]. Nevertheless, these trends are dynamic, given changes in the prevalence of risk factors and uncertainties about the impact of enhanced HCV treatment uptake $[6,30]$, and should be closely monitored.

This study has several limitations. First, in Australia, HBV notifications are based on evidence of chronic infection. However, the number of people with active HBV replication could not be evaluated. Second, HCV diagnosis for surveillance does not necessitate HCV RNA confirmation, and is commonly based on anti-HCV antibody detection. Therefore, an estimated $25 \%$ of people with an HCV notification would have spontaneously cleared their HCV infection. Nevertheless, these limitations are not thought to have a significant impact on the findings of this study, given fixed surveillance definition and systems in NSW during the study period. Third, in the presence of multiple chronic diseases that are each potentially fatal, the decision about selecting the underlying cause of death can be subjective; however, where possible (i.e. liver-related mortality), a multiple cause definition was used to include important cause information that might have been overlooked otherwise [11]. Fourth, in evaluation of the association between risk factors and liver-related mortality, a sensitivity analysis was performed using Fine and Gray regression, to account for competing risks. Period of HBV notification was not associated with liver-related mortality in the adjusted Fine and Gray model. This difference could be due to the potential impact of higher numbers of deaths among older people who had an HBV notification in recent time periods, and inclusion of these deaths in the risk set of a competing risk framework. Fifth, using administrative data to assess risk factors for $\mathrm{HBV}$ - and $\mathrm{HCV}$ related liver mortality has clear limitations, including lack of sociodemographic and health risk information such as smoking, poor diet, physical inactivity, mental health problems, and low income. Sixth, DC-related hospitalisation and mortality were defined by a limited number of conditions that appear to be strong indicators of the decompensated stage of cirrhosis; however, this definition has not been validated against the clinical diagnosis of DC, and requires further validation studies. Finally, given lack of individual-level treatment data, in the analyses of factors associated with liver-related mortality, it was not possible to determine which associated factors were proxies for not receiving antiviral therapy. During the era of interferon-containing $\mathrm{HCV}$ treatments, factors including older age, advanced liver disease, and history of alcohol use could have been associated with lower $\mathrm{HCV}$ treatment uptake, given poorer response rates [31].

\section{Conclusions}

In conclusion, this population-level data linkage study provides evidence for contrasting trends in HBV- and HCV-related liver mortality in NSW, Australia, a possible result of differences in antiviral therapy efficacy and uptake. These findings also suggest action against health risk behaviours should form a strong component of HBV and HCV public health strategies. Use of administrative databases for surveillance, particularly with the addition of individual-level antiviral treatment data will be a valuable tool for public health research and evaluation of improved strategies for viral hepatitis diagnosis and treatment in the future. 


\section{Additional file}

Additional file 1: Table S1. ICD-10 codes used to define cause-specific mortality and hospital admissions among people with an HBV and HCV notification, NSW 1993-2012, $n=150,403$. Table S2. Cause-specific mortality among people with an HBV and HCV notification, NSW 1993-2012, by ICD-10 chapter, $n=150,403$. Table S3. Unadjusted analysis of factors associated with liver-related mortality among people with an HBV notification, NSW 1993-2012, $n=57,929$. Table S4. Unadjusted analysis of factors associated with liver-related mortality among people with an HCV notification, NSW 1993-2012, $n=96,250$. Table S5. Other cause-related mortality among people with an HBV and HCV notification, NSW 1993-2012, by ICD-10 chapter and year of birth, $n=150,403$. Table S6. Adjusted analysis of factors associated with liver-related mortality among people with an HBV and HCV notification, NSW 1993-2012, $n=150,403$. (DOCX 50 kb)

\section{Abbreviations}

APDC: Admitted patient data collection; AUD: Alcohol-use disorder; COD URF: Cause of death unit record file; DAA: Direct-acting antiviral; DC: Decompensated cirrhosis; ESLD: End-stage liver disease; HBV: Hepatitis B virus; HCC: Hepatocellular carcinoma; HCV: Hepatitis C virus; ICD-10: International classification of diseases and related health problems; NCIMS: Information management system; NHR: National HIV Registry; NSW: New South Wales; OST: Opioid substitution therapy; PHDAS: Pharmaceutical drugs of addiction system; RBDM: Registry of births, deaths and marriages; WHO: World Health Organization

\section{Funding}

The Kirby Institute is funded by the Australian Government Department of Health, under the agreement ID number 2-D3X513. This publication is part of the Bloodborne viruses and sexually transmissible infections Research, Strategic Interventions and Evaluation (BRISE) program, funded by the NSW Ministry of Health. This publication has received funding from the Sydney West Translational Cancer Research Centre (SW-TCRC) program "HOTTer-West: HCC Outcome improvements Through Translational research in WESTern Sydney". MA is the recipient of a CASCADE International Fellowship. The research leading to these results has received funding from the People Programme (Marie Curie Actions) of the European Union's Seventh Framework Programme (FP7/2007-2013) under REA grant agreement No PCOFUND-GA-2012-600181. J Grebely is supported by a National Health and Medical Research Council of (NHMRC) Australia Career Development Fellowship. BH is supported by an NHMRC of Australia Early Career Fellowship. J George is supported by an NHMRC of Australia Program Grant (1053206), project grants (632630, 1049857), a Sydney West Translational Cancer Research Centre grant funded by the Cancer Institute NSW, and the Robert W. Storr Bequest to the Sydney Medical Foundation, University of Sydney. LD is supported by an NHMRC Australia Principal Research Fellowship (1071742). GD is supported through an NHMRC of Australia Practitioner Fellowships.

\section{Availability of data and materials}

This publication has used highly sensitive health information through linkage of several administrative datasets. De-identified linked information has been provided to the research team under strict privacy regulations, outlined by the Health Records and Information Privacy Act 2002 (NSW). Except in the form of conclusions drawn from the data, researchers do not have permission to disclose any data to any person other than those authorised for the research project.

\section{Authors' contributions}

MA, JGrebely, and GD contributed to study conception and design, data acquisition and analysis, interpretation of findings, and drafting of the manuscript; $\mathrm{BH}, \mathrm{JA}, \mathrm{ML}$, and JGeorge contributed to study conception and design, data acquisition and analysis, and interpretation of findings, and; SL and LD contributed to study conception and design, and interpretation of findings. All authors have read and approved the manuscript.

\section{Ethics approval and consent to participate}

This publication has complete approval from the NSW Population \& Health Services Research Ethics Committee (Cancer Institute NSW reference number: 2013/11/493). The Ethics Committee granted a waiver of the usual requirement for the consent of the individual to the use of their health information in a research project, in line with the State Privacy Commissioner's Guidelines for Research and the Health Records and Information Privacy Act 2002 (NSW) and the Guidelines approved under Section 95 of the Privacy Act 1988.

\section{Competing interests}

J Grebely has received research support and is a consultant for Abbvie, Gilead Sciences and Merck. J Grebely has received research support from Bristol-Myers Squibb and Cepheid. ML has received research support from Merck, Bristol-Myers Squibb, Boehringer Ingelheim, Janssen-Cilag, Gilead Sciences, and ViiV HealthCare. ML has received consultancy and workshop fees from Gilead Sciences. ML has received Data Safety Monitoring Board Committee fees from Sirtex Pty Ltd. J George is on the speaker's bureau for Gilead Sciences, Merck, Janssen, Roche, and Pharmaxis. J George is a member of advisory board for Gilead Sciences, Merck, Janssen, Bristol-Myers Squibb, Abbvie, Roche, GlaxoSmithKline, and Pharmaxis. J George has received travel support from Gilead Sciences, Merck, Bristol-Myers Squibb, Abbvie, and Roche. SL and LD have received untied educational grant funding from Indivior to examine naloxone for opioid overdose reversal (2015-2017), and OST use among chronic pain patients (2015-2016). GD has received research support and is a consultant for Gilead Sciences, Merck, and Janssen. GD has received research support from Bristol-Myers Squibb, Abbvie, and Roche. $\mathrm{GD}$ is on the speaker's bureau for Gilead Sciences, Merck, Janssen, and Roche. GD is a member of advisory board for Gilead Sciences, Merck, Janssen, Bristol-Myers Squibb, Abbvie, Roche, GlaxoSmithKline, and Abbott Diagnostics. GD has received travel support from Gilead Sciences, Merck, Bristol-Myers Squibb, Abbvie, and Roche. MA and BH are members of the BMC Infectious Diseases editorial board. Other authors have no commercial relationships that might pose a conflict of interest in connection with this manuscript.

\section{Publisher's Note}

Springer Nature remains neutral with regard to jurisdictional claims in published maps and institutional affiliations.

\section{Author details}

${ }^{1}$ Biostatistics and Databases Program, The Kirby Institute, UNSW Sydney, Wallace Wurth Building, UNSW, Sydney, NSW 2052, Australia. ²Department of Health Systems and Populations, Macquarie University, Sydney, NSW, Australia. ${ }^{3}$ National Drug and Alcohol Research Centre, UNSW Sydney, Sydney, NSW, Australia. ${ }^{4}$ Storr Liver Centre, Westmead Millennium Institute, University of Sydney and Westmead Hospital, Westmead, NSW, Australia.

Received: 6 July 2017 Accepted: 24 April 2018

Published online: 09 May 2018

References

1. Stanaway JD, Flaxman AD, Naghavi M, Fitzmaurice C, Vos T, Abubakar I, et al. The global burden of viral hepatitis from 1990 to 2013: findings from the global burden of disease study 2013. Lancet. 2016;388(10049):1081-8.

2. Dore G, Ward J, Thursz M. Hepatitis C disease burden and strategies to manage the burden (guest editors mark Thursz, Gregory dore and John Ward). J Viral Hepat. 2014;21(S1):1-4.

3. World Health Organization. Global health sector strategy on viral hepatitis 2016-2021. 2016. Available from: www.who.int/hepatitis/strategy2016-2021/ ghss-hep/en/. Feb 2018.

4. Degenhardt L, Hall W. Extent of illicit drug use and dependence, and their contribution to the global burden of disease. Lancet. 2012;379(9810):55-70.

5. Younossi Z, Zheng L, Stepanova M, Venkatesan C, Mir H. Moderate, excessive or heavy alcohol consumption: each is significantly associated with increased mortality in patients with chronic hepatitis C. Aliment Pharmacol Ther. 2013;37(7):703-9.

6. Petta S, Maida M, Macaluso FS, Barbara M, Licata A, Craxì A, et al. Hepatitis C virus infection is associated with increased cardiovascular mortality: a meta-analysis of observational studies. Gastroenterology. 2016;150(1):145-55.

7. Lozano R, Naghavi M, Foreman K, Lim S, Shibuya K, Aboyans V, et al. Global and regional mortality from 235 causes of death for 20 age groups in 1990 and 2010: a systematic analysis for the global burden of disease study 2010. Lancet. 2013;380(9859):2095-128. 
8. Jacobson IM, Davis GL, El-Serag H, Negro F, Trépo C. Prevalence and challenges of liver diseases in patients with chronic hepatitis $C$ virus infection. Clin Gastroenterol Hepatol. 2010;8(11):924-33.

9. The Centre for Health Record Linkage (CHeReL). 2017. Available from: http://www.cherel.org.au/. Accessed Dec 2017.

10. McDonald AM, Crofts N, Blumer CE, Gertig DM, Patten JJ, Roberts M, et al. The pattern of diagnosed HIV infection in Australia, 1984-1992. AIDS 1994;8(4):513-9.

11. Australian Institute of Health and Welfare. Multiple causes of death. An analysis of all natural and selected chronic disease causes of death 1997-2007. Bulletin no. 105. Cat. No. AUS 159. Canberra: AlHW; 2012. Available from: http://www.aihw.gov.au/publication-detail/?id=10737422603. December 2017.

12. Friedmann PD. Alcohol use in adults. N Engl J Med. 2013;368(4):365-73.

13. Törner A, Duberg A-S, Dickman P, Svensson Å. A proposed method to adjust for selection bias in cohort studies. Am J Epidemiol. 2010;171(5):602-8.

14. Wong GLH, Chan HLY, Mak CWH, Lee SKY, Ip ZMY, Lam ATH, et al. Entecavir treatment reduces hepatic events and deaths in chronic hepatitis $B$ patients with liver cirrhosis. Hepatology. 2013;58(5):1537-47.

15. Papatheodoridis GV, Chan HL-Y, Hansen BE, Janssen HL, Lampertico P. Risk of hepatocellular carcinoma in chronic hepatitis B: assessment and modification with current antiviral therapy. J Hepatol. 2015;62(4):956-67.

16. The Kirby Institute. Annual surveillance report of HIV, viral hepatitis, STIs, 2011: The Kirby Institute, UNSW Sydney; 2017. Available from: https://kirby. unsw.edu.au/report/annual-surveillance-report-hiv-viral-hepatitis-stis-2011. Accessed Dec 2017.

17. Allard NL, MacLachlan JH, Cowie BC. The cascade of care for Australians living with chronic hepatitis B: measuring access to diagnosis, management and treatment. Aust N Z J Public Health. 2015;39(3):255-9.

18. Hajarizadeh B, Grebely J, McManus H, Estes C, Razavi H, Gray RT, et al. Chronic hepatitis C burden and care cascade in Australia in the era of interferon-based treatment. J Gastroenterol Hepatol. 2017;32(1):229-36.

19. Dore GJ, Hajarizadeh B. Elimination of hepatitis C virus in Australia laying the foundation. Infect Dis Clin N Am. 2018; in press

20. Zhou K, Fitzpatrick T, Walsh N, Kim JY, Chou R, Lackey M, et al. Interventions to optimise the care continuum for chronic viral hepatitis: a systematic review and meta-analyses. Lancet Infect Dis. 2016;16(12):1409-22.

21. Hajarizadeh B, Grebely J, Dore GJ. Epidemiology and natural history of HCV infection. Nat Rev Gastroenterol Hepatol. 2013;10(9):553-62.

22. Fattovich G, Bortolotti F, Donato F. Natural history of chronic hepatitis B: special emphasis on disease progression and prognostic factors. J Hepatol. 2008;48(2):335-52.

23. Tsochatzis EA, Bosch J, Burroughs AK. Liver cirrhosis. Lancet. 2014;383(9930):1749-61.

24. Degenhardt L, Roxburgh A, Black E, Bruno R. The epidemiology of methamphetamine use and harm in Australia. Drug Alcohol Rev. 2008;27(3):243-52.

25. Horyniak D, Stoové M, Degenhardt L, Aitken C, Kerr T, Dietze P. How do drug market changes affect characteristics of injecting initiation and subsequent patterns of drug use? Findings from a cohort of regular heroin and methamphetamine injectors in Melbourne, Australia. Int J Drug Policy. 2015;26(1):43-50.

26. Blanch B, Pearson SA, Haber PS. An overview of the patterns of prescription opioid use, costs and related harms in Australia. Br J Clin Pharmacol. 2014;78(5):1159-66.

27. Stafford J, Breen C. Australian drug trends 2015. Findings from the illicit drug reporting system (IDRS). Australian drug trend series. No. 145. Sydney: National Drug and Alcohol Research Centre, UNSW Sydney; 2017. Available from: https://ndarc.med.unsw.edu.au/sites/default/files/ndarc/resources/ AUSTRALIAN_IDRS_2015_0.pdf.

28. Charlson FJ, Baxter AJ, Dua T, Degenhardt L, Whiteford HA, Vos T. Excess mortality from mental, neurological and substance use disorders in the global burden of disease study 2010. Epidemiol Psychiatr Sci. 2015;24(02):121-40.

29. O'Flaherty M, Buchan I, Capewell S. Contributions of treatment and lifestyle to declining CVD mortality: why have CVD mortality rates declined so much since the 1960s? Heart. 2013;99(3):159-62.
30. O'Flaherty M, Allender S, Taylor R, Stevenson C, Peeters A, Capewell S. The decline in coronary heart disease mortality is slowing in young adults (Australia 1976-2006): a time trend analysis. Int J Cardiol. 2012;158(2):193-8.

31. El-Serag HB, Kanwal F, Richardson P, Kramer J. Risk of hepatocellular carcinoma after sustained virological response in veterans with hepatitis $C$ virus infection. Hepatology. 2016;64(1):130-7.

\section{Ready to submit your research? Choose BMC and benefit from:}

- fast, convenient online submission

- thorough peer review by experienced researchers in your field

- rapid publication on acceptance

- support for research data, including large and complex data types

- gold Open Access which fosters wider collaboration and increased citations

- maximum visibility for your research: over $100 \mathrm{M}$ website views per year

At BMC, research is always in progress.

Learn more biomedcentral.com/submissions 